\title{
On the scaling of waiting-time distributions of the negative IMF $B_{z}$ component
}

\author{
R. D’Amicis ${ }^{1}$, R. Bruno ${ }^{1}$, B. Bavassano ${ }^{1}$, V. Carbone ${ }^{2}$, and L. Sorriso-Valvo ${ }^{3}$ \\ ${ }^{1}$ Istituto di Fisica dello Spazio Interplanetario (INAF), 00133 Roma, Italy \\ ${ }^{2}$ Dipartimento di Fisica Università della Calabria, 87036 Rende (Cs), Italy \\ ${ }^{3}$ Liquid Crystal Laboratory, INFM/CNR, 87030 Rende (Cs), Italy
}

Received: 19 January 2006 - Revised: 4 August 2006 - Accepted: 29 August 2006 - Published: 20 October 2006

\begin{abstract}
Statistics associated with the fluctuations in solar wind parameters show a remarkable dependence on the solar activity phase. In particular, we focus our attention on the waiting-time statistics governing the MHD fluctuations of the z-component of the interplanetary magnetic field, which are important within the framework of the Sun-Earth connections, and briefly discuss the preliminary results. Data from several spacecrafts, covering different phases of the solar cycle and different radial distances, are used. We found that propagating Alfvénic fluctuations and convected structures strongly influence the statistics which vary from quasiPoissonian to power law.
\end{abstract}

Keywords. Interplanetary physics (Interplanetary magnetic fields; MHD waves and turbulence; Solar wind plasma)

\section{Introduction}

The Sun-Earth system is a highly dynamical system in which a continuous exchange of mass, energy and momentum occurs between the solar wind and the Earth's magnetosphere through reconnection processes (Vasyliunas, 1975). However, the understanding of the magnetospheric response to the solar wind variations is still an open problem, as it involves different mechanisms of energy release and multiscale coupling phenomena.

Recent studies have focused on the comparison between the coupling parameters, like $\epsilon$ and $v B_{s}$, and auroral indices, to ascertain wether, to the lowest order, the magnetospheric response is directly connected to the solar wind's behaviour. In particular, Hnat et al. $(2002,2003)$ characterised probability distribution functions (hereafter PDFs) of the fluctuations of $\epsilon$ parameter and those of the auroral indices, finding scaling laws in the peaks of the distributions at differ-

Correspondence to: R. D'Amicis

(damicis@ifsi.rm.cnr.it) ent scales similar for the solar wind driver and the magnetospheric response. Moreover, Freeman et al. (2000a) investigated the similarities and differences of burst lifetime distributions finding that $\mathrm{AL}$ and $\mathrm{AU}$ lifetime distributions, have the same power law of the coupling parameters $\left(v B_{s}\right.$ and $\left.\epsilon\right)$. Freeman et al. (2000b) analysed the waiting-time distribution of the Poynting vector, finding that for two decades a power law has described such distribution.

In the present work we intend to study waiting-time statistics of north-south turnings of the IMF, i.e. the time interval between the end of a magnetic turning and the beginning of the next one, in order to emphasize the possible correlations between these events. By studying waiting time statistics it is possible to have information on the process we are analysing. For a turbulent process, we expect that waiting-time distributions follow a power law (Boffetta et al., 1999; Bruno and Carbone, 2005). In fact, as turbulence may be viewed as a fragmentation process that carries energy from large to small scales, there exists a strong correlation between structures generated at different scales. On the contrary, for a stochastic process, events are not correlated and consequently, we expect a Poissonian distribution for waiting times.

In addition, we must take into account that the solar wind shows an intermittent behaviour (Marsch and Liu, 1993). This means that the shape of the PDFs of the solar wind fluctuations, at a given scale, depends on the scale considered. In fact, it roughly evolves from a Gaussian, at large scales, to a stretched exponential at small scales, as the largest events, contained in the tails of the distribution, show a much higher probability. The largest events are associated with convected structures that sporadically form in the turbulent medium (see Frisch, 1995, for a general discussion on intermittency or Bruno and Carbone, 2005, for recent results in the solar wind context). Previous studies regarding crossing time analysis and waiting-time statistics have been done not taking into account that the solar wind shows a different intermittent behaviour for different phases of the solar cycle (Vörös

Published by Copernicus GmbH on behalf of the European Geosciences Union. 


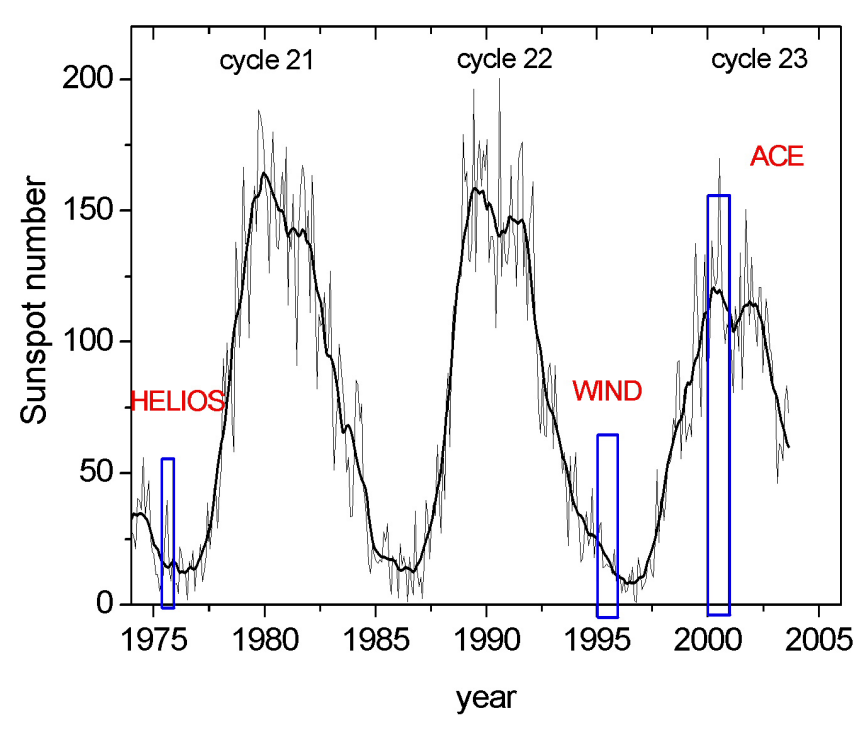

Fig. 1. Daily sunspot number as a function of time and its monthly average (smoothed curve). The boxes show the selected time intervals.

et al., 2002; D'Amicis et al., 2004; Hnat et al., 2005). As a matter of fact, the probability of finding extreme events is higher at solar maximum, given the higher variability of the parameters that characterize the solar wind.

Consequently, in this paper we will focus on a comparative study of $B_{S}$ waiting times for different phases of the solar cycle as an introductory work to future study on solar windmagnetosphere coupling.

\section{Data selection}

The present analysis was performed using data recorded by the magnetic field instruments (MFI) on board WIND and ACE satellites in GSE coordinates, which correspond to a period of minimum and maximum in the solar activity, respectively. Data are available at a time resolution of $16 \mathrm{~s}$ and $3 \mathrm{~s}$, for WIND and ACE, respectively. However, the two satellites follow different trajectories. In fact, ACE is positioned at the L1 point, while WIND has a more complicated orbit that crosses the magnetosphere. As we are interested only in solar wind data, we selected only those periods when WIND was in the interplanetary space.

In order to have the same time resolution for the two magnetic field data sets, we performed averages at $48 \mathrm{~s}$, a value which is a multiple of both time resolutions. We selected periods of time lasting 16 months (about 900000 points) for both the minimum (from January 1995 to April 1996) and maximum of the solar cycle (from May 2000 to August 2001), as shown in Fig. 1.

In the second part of this paper, in order to support the interpretation we give the results obtained in the data analysis
Table 1. HELIOS data: fast and slow streams.

\begin{tabular}{ccc}
\hline $\begin{array}{l}\text { time interval } \\
\text { (day of year) }\end{array}$ & $\begin{array}{c}\text { distance } \\
(\mathrm{AU})\end{array}$ & $\begin{array}{c}<\mathrm{V}> \\
(\mathrm{km} / \mathrm{s})\end{array}$ \\
\hline $105.63-108.13$ & 0.3 & 730 \\
$99.50-102.00$ & 0.3 & 400 \\
$49.25-51.75$ & 0.9 & 650 \\
$45.38-47.88$ & 0.9 & 430 \\
\hline
\end{tabular}

performed at $1 \mathrm{AU}$, we used data from the Helios 2 mission, during its primary mission to the Sun in 1976 (see Sect. 4). The time interval selected is a period of minimum solar activity, characterized by the stream structure of the solar wind. In addition, Helios' orbit allows one to investigate radial dependences. In fact, it is the only data set available covering heliocentric distances between $0.29 \mathrm{AU}$, the closest approach to the Sun so far and $1 \mathrm{AU}$. In this range, we selected fast and slow streams, as indicated in Table 1. The data time resolution is $6 \mathrm{~s}$. Since every stream selected lasts for about 2.5 days, each period is characterized by about 14400 points.

\section{Waiting time dependence on solar cycle}

The aim of the present study is to investigate waiting-time statistics of $B_{s}$ as a function of the solar cycle. The waiting time is defined as the time spent by a generic measurement $x(t)$ below or above a given threshold value. As far as $B_{S}$ is concerned, it coincides with the duration of time for which $B_{z}$ is positive.

Power law scaling with an exponential cutoff $\left(T_{c}\right)$

$y(t)=A t^{-\alpha} \exp \left(-t / T_{c}\right)$

seems to be appropriate to describe the behavior of solar wind waiting-time statistics, as it takes into account both contributions: the one associated with long-term correlations, described by the power law, and the one related to uncorrelated events, described by the Poissonian distribution. In Eq. (1), A, $\alpha$ and $T_{c}$ are parameters to be determined by the fitting procedure. Freeman et al. (2000a) proved that the exponential truncation of the power law is a function of the threshold used for this kind of analysis. In the present paper, however, the choice of the threshold is not arbitrary, and it is set to 0 because of the definition of $B_{s}$ itself.

Figure 2 displays the waiting time distributions of $B_{s}$ at solar minimum and maximum (left- and right-hand side panels, respectively), in which the points are derived from an average of the histogram over logarithmically, equally spaced intervals and is normalized to the maximum value. We chose this kind of normalization since we are simply interested in discussing the shape of the distributions. 


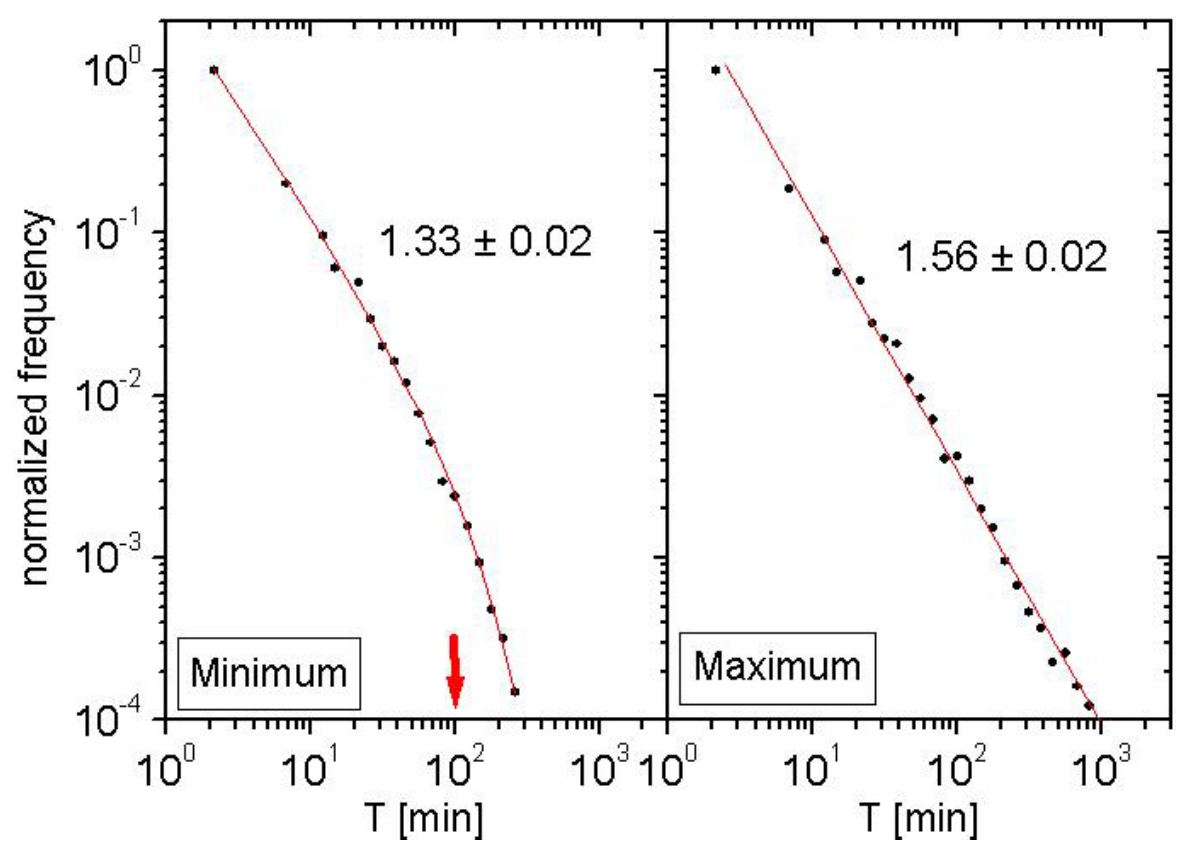

Fig. 2. Waiting time distributions of $B_{S}$ at solar minimum and maximum on the left- and right-hand side panels, respectively. The points are derived from an average of the histogram over logarithmically, equally spaced intervals and are normalized to the maximum value. The solid lines refer to the fits computed by Eq. (1). The red arrow, in the left panel, indicates the position of the cutoff. The slope of the power law is indicated in the picture.

Results relative to solar minimum, shown on the left-hand side of Fig. 2, suggest that both contributions, the one associated with the power law and the one described by the Poissonian, play a role. As a matter of fact, $B_{s}$ events follow a power law distribution $(\alpha=1.33)$ as far as a time lag of about $100 \mathrm{~min}$, where the presence of a cutoff (whose position is indicated by the red arrow in Fig. 2) suggests the loss of long-range correlations.

These results are in agreement with the ones found by Freeman et al. (2000a). In fact, these authors showed that both $v B_{s}$ and $\epsilon$ burst lifetime distributions can be described by a power law with an exponential cutoff. Although the time interval we analyzed does not exactly match the one in Freeman et al. (2000a), as they chose a time window that also included the rising phase of the solar cycle, the results we find are similar. In fact, we find that the power law exponent found at solar minimum in our work is similar to the one computed for $\epsilon$ burst lifetime distributions $(\alpha=1.35)$, as shown in Freeman et al. (2000a).

On the contrary, at solar maximum (right-hand side of Fig. 2), we found that a simple power law $(\alpha=1.56)$ is sufficient to describe the trend of the distribution over the whole time interval considered suggesting that $T_{c} \rightarrow \infty$. The values of the parameters determined by the fitting procedure are shown in Table 2. Freeman et al. (2000b) found a similar slope in the power law of the waiting-time distribution of the Poynting vector $(\alpha=1.67)$.
Table 2. Parameters of the fit: maximum and minimum comparison. $T_{c}$ is expressed in min.

\begin{tabular}{ccc}
\hline parameters & minimum & maximum \\
\hline $\mathrm{A}$ & $2.82 \pm 0.08$ & $0.65 \pm 0.05$ \\
$\alpha$ & $1.33 \pm 0.02$ & $1.56 \pm 0.02$ \\
$T_{c}$ & $105 \pm 20$ & - \\
\hline
\end{tabular}

The comparison made between our work and previous work obviously shows that crossing and waiting-time analysis computed over interplanetary parameters, such as $B_{s}$, $v B_{s}$ and $\epsilon$, lead to similar results (power law distributions with slopes always $<2$ ). However, it is worth emphasizing that the presence of the exponential cutoff is a characteristic of solar minimum activity.

We can explain this result, taking into account the fact that during solar minimum, the presence of fast corotating streams, largely populated by Alfvénic fluctuations, is stronger than during solar maximum (Tu and Marsch, 1993). Consequently, we expect that during solar minimum fluctuations are more stochastic with a consequent loss of long time correlations. This conclusion is also supported by the fact that, at solar minimum, the cutoff is around the typical period of the Alfvénic fluctuations (Bruno et al., 1985; Marsch and Tu, 1990). 


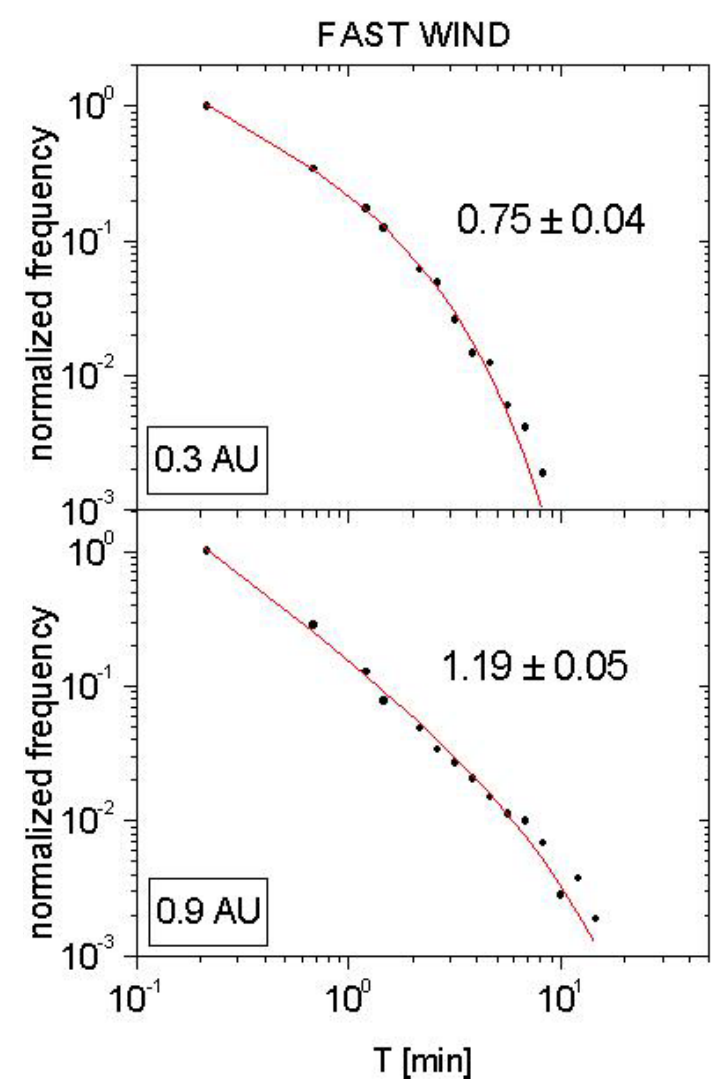

Fig. 3. Waiting time distributions of $B_{S}$ for fast wind at different radial distances. The slope of the power law is indicated in the picture. The points are derived from an average of the histogram over logarithmically equally spaced intervals and are normalized to the maximum value.

Table 3. Parameters of the fit: fast wind. $T_{C}$ is expressed in min.

\begin{tabular}{ccc}
\hline parameters & $0.3 \mathrm{AU}$ & $0.9 \mathrm{AU}$ \\
\hline $\mathrm{A}$ & $0.38 \pm 0.01$ & $0.17 \pm 0.01$ \\
$\alpha$ & $0.75 \pm 0.04$ & $1.19 \pm 0.05$ \\
$T_{c}$ & $1.76 \pm 0.09$ & $8 \pm 2$ \\
\hline
\end{tabular}

\section{Waiting-time dependence on heliocentric distance}

To corroborate this idea, we consider IMF data recorded by Helios 2 at different solar wind velocity and different distances, as shown in Table 1. In fact, we have the possibility to analyse high and low velocity periods that are well known in literature. In particular, the time intervals, referring to fast wind, correspond to the same stream which is observed after two solar rotations (Bavassano et al., 1982). Moreover, Bruno et al. (1985), studying the same intervals, have shown that Alfvénicity rapidly evolves with heliocentric distances, being larger at $0.3 \mathrm{AU}$ than at $1 \mathrm{AU}$. We can compare the waiting time distribution of those periods to the waiting time

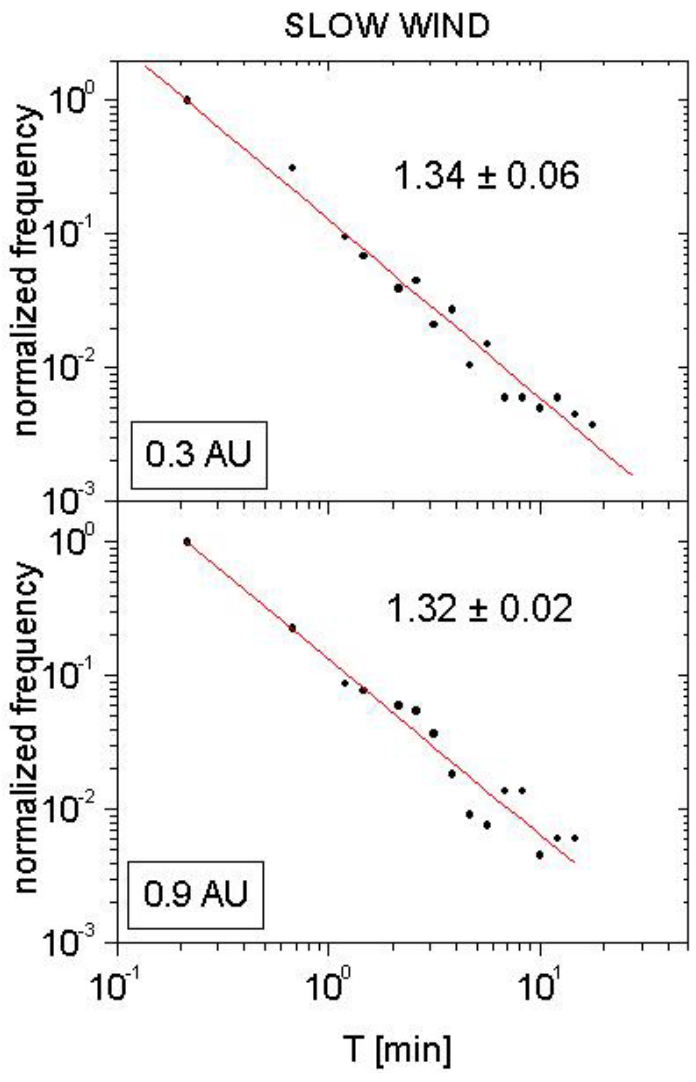

Fig. 4. Waiting-time distributions of $B_{S}$ for slow wind at different radial distances. The slope of the power law is indicated in the picture. The points are derived from an average of the histogram over logarithmically, equally spaced intervals and are normalized to the maximum value.

Table 4. Parameters of the fit: slow wind.

\begin{tabular}{ccc}
\hline parameters & $0.3 \mathrm{AU}$ & $0.9 \mathrm{AU}$ \\
\hline $\mathrm{A}$ & $0.90 \pm 0.05$ & $0.89 \pm 0.06$ \\
$\alpha$ & $1.34 \pm 0.06$ & $1.32 \pm 0.02$ \\
\hline
\end{tabular}

distribution obtained within low velocity and less Afvénic solar wind samples.

Figure 3 shows fast wind waiting time distributions at different radial distances. The points are derived from an average of the histogram over logarithmically, equally spaced intervals and are normalized to the maximum value. From top to bottom, we notice a radial evolution of waiting time statistics. In fact, at $0.3 \mathrm{AU}$ the distribution is nearly a Poissonian, with a cutoff (whose position is indicated by the red arrow in Fig. 3) of the order of a few minutes. At $0.9 \mathrm{AU}$ we notice that the distribution, fitted by the same Eq. (1), is more stretched. In fact, we find a higher slope $(\alpha=1.19)$ and a higher $T_{c}(8 \mathrm{~min})$ than the corresponding values found at 


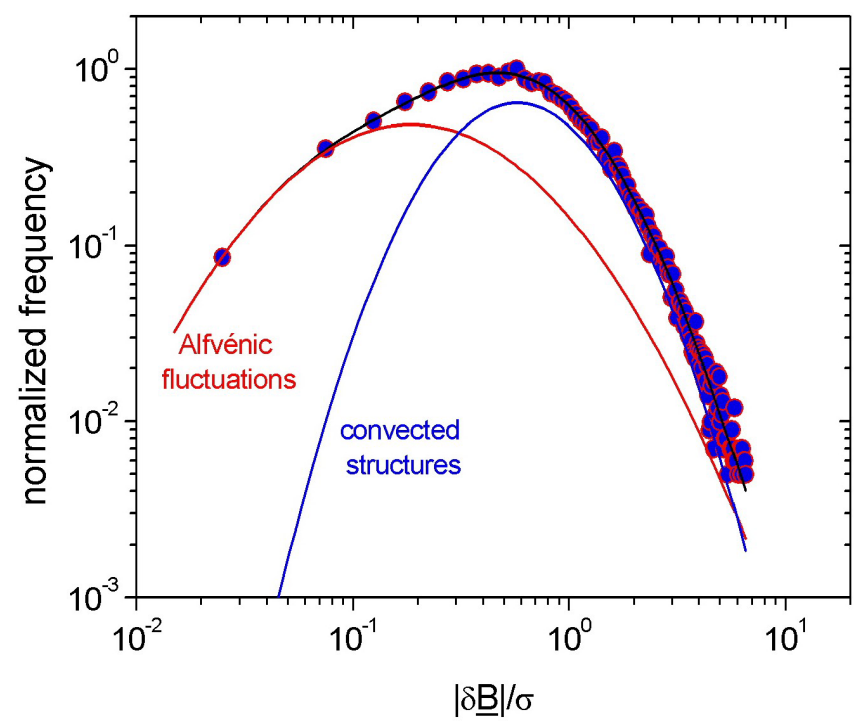

Fig. 5. $|\delta \boldsymbol{B}(t)|$ distribution in $\sigma$ units described by two lognormal components corresponding to Alfvénic fluctuations and convected structures, respectively (adapted from Bruno et al., 2004).

$0.3 \mathrm{AU}\left(\alpha=0.75\right.$ and $\left.T_{c} 1.76 \mathrm{~min}\right)$. The parameters of the fit are displayed in Table 3.

The previous results are in agreement with the fact that Alfvénicity evolves as a function of the heliocentric distance (Bruno et al., 1985). In fact, at $0.3 \mathrm{AU}$ long time correlations are nearly lost while they emerge at larger radial distances as the Alfvénic fluctuations are gradually depleted.

On the contrary, Fig. 4 shows waiting times for slow wind. It is clear that, at the heliocentric distances considered, the distributions are power laws and, within the errors, they have the same slopes, as indicated in Table 4. This suggests not only the existence of long time correlations in $B_{s}$ events but also the persistance of the same statistics as far as $1 \mathrm{AU}$.

\section{Numerical simulations}

To reproduce interplanetary data, only from a statistical point of view, we refer to the work of Bruno et al. (2004). These authors used Helios' data at a time resolution of $6 \mathrm{~s}$ and developed a toy model to reproduce data by a random walk process governed by a double lognormal statistics. In fact, by studying the probability distributions of $|\delta \boldsymbol{B}(\boldsymbol{t})|$, defined as follows:

$$
|\delta \boldsymbol{B}(t)|=\sqrt{\sum_{i=x, y, z}\left(B_{i}(t)-B_{i}\left(t_{0}\right)\right)^{2}},
$$

where $\boldsymbol{B}\left(t_{0}\right)$ is the direction of the first vector of $\boldsymbol{B}(t)$, they found that the $|\delta \boldsymbol{B}(\boldsymbol{t})|$ distribution is described by two lognormal components (as shown in Fig. 5), which are present in both fast and slow winds, even with a different statistical

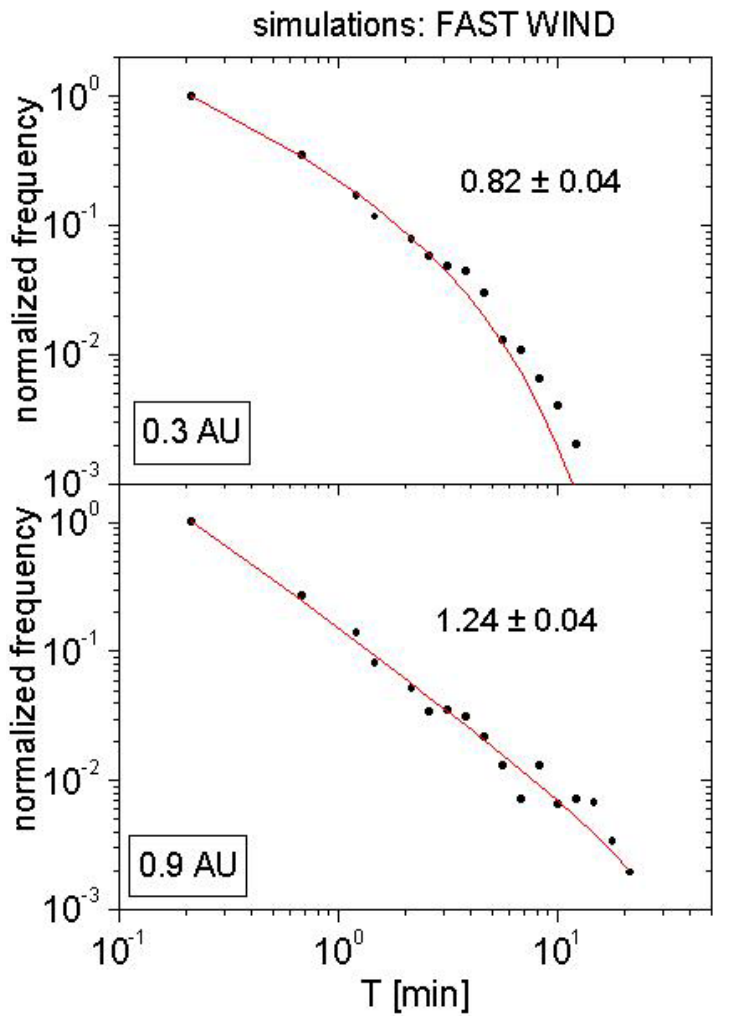

Fig. 6. Fast wind numerical simulation: waiting-time distributions as a function of radial distance. The points are derived from an average of the histogram over logarithmically, equally spaced intervals and are normalized to the maximum value.

weight. While in a fast wind distribution, the two components have comparable weights, in slow wind, one of the two has a weight much greater than the other. Moreover, in a fast wind, Bruno et al. (2004) noticed a radial evolution of the two components, not observed in slow wind. Those authors interpreted their results, taking into account that the component which describes the small $|\delta \boldsymbol{B}(t)| / \sigma$ (the one which evolves radially) represents the contribution due to Alfvénic fluctuations, while the other one represents convected structures.

We used the same procedure of Bruno et al. (2004) to generate an artificial time series. In particular, we used the same distributions found by Bruno et al. (2004) at 0.3 and $0.9 \mathrm{AU}$ for both fast and slow winds to produce a corresponding time series of about 80000 records each. Each record contains the three cartesian components of the position of the tip of a unitary vector which is free floating on the surface of a sphere. The vector difference $|\delta \boldsymbol{B}|$ between the two successive orientations of the unitary vector is randomly extracted from the relative distribution. In this way we obtain a random walk on the surface of the sphere whose movements obey double lognormal statistics.

Figure 6 shows the waiting-time distributions obtained from simulated data at different radial distances in which one 


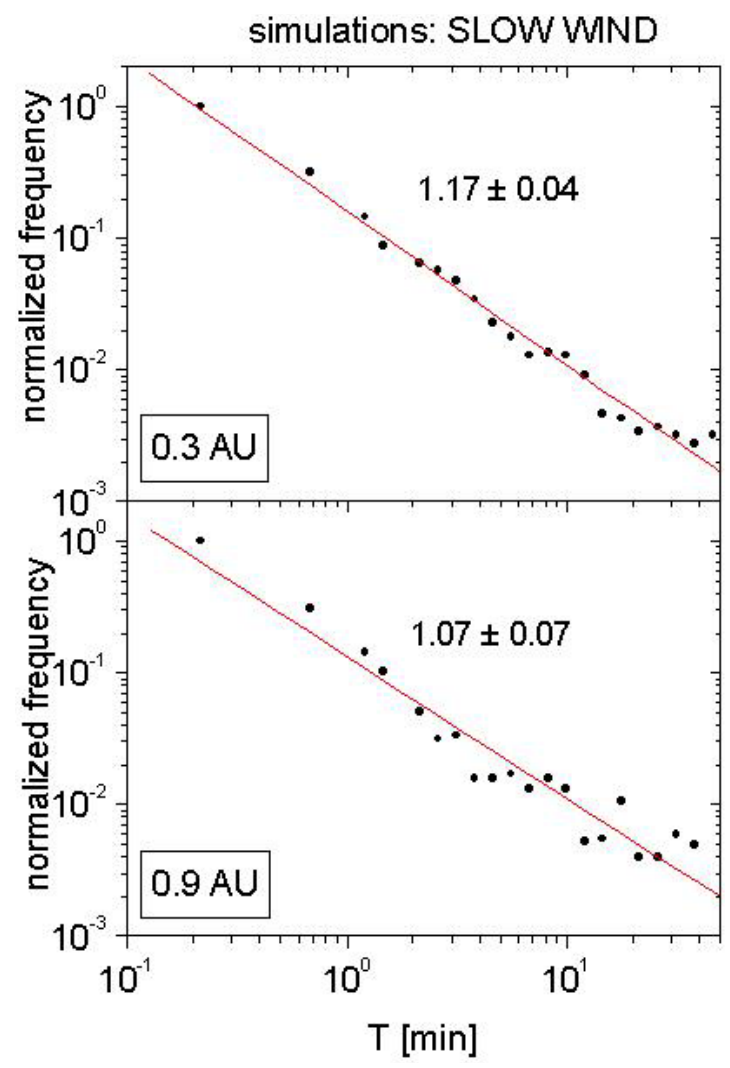

Fig. 7. Slow wind numerical simulation: waiting-time distributions as a function of the radial distance. The points are derived from an average of the histogram over logarithmically, equally spaced intervals and are normalized to the maximum value.

can observe a radial evolution of the statistics. As a matter of fact, $\alpha$ and $T_{c}$ increase from $\alpha=0.82$ and $T_{c}=3 \mathrm{~min}$ at $0.3 \mathrm{AU}$ to $\alpha=1.24$ and $T_{c}=38 \mathrm{~min}$ at $0.9 \mathrm{AU}$. Although we find the same power law exponents both in Helios' data and in simulated ones, within the errors (compare Fig. 3 and 6), our findings concerning the exponential cutoff are different, especially at $0.9 \mathrm{AU}$.

The results concerning simulated slow wind are shown in Fig. 7, which displays waiting-time distributions at 0.3 and 0.9 AU. We notice that both distributions are power laws and they have, within the errors, the same slope. The power law exponents found in the artificial data are slightly different from the ones from the Helios' data (see Fig. 4 and 7 for comparison).

The artificial data we obtained from this procedure reproduce qualitatively rather than quantitatively the results we had from the analysis of Helios' experimental data. However, we are more interested in the trend of the waiting time distributions rather than in comparing the numerical values of the parameters evaluated by the fitting procedures. As a matter of fact, our model allows us to reproduce the slope of the distributions both at solar minimum and maximum. In fact, at solar minimum, the distributions are described by a power law with exponential cutoff. Moreover, we observe that these distributions evolve as a function of the radial distance similar to the results found for the real data. For what concerns solar maximum, on the contrary, we observe power law distributions which do not evolve radially.

\section{Summary and conclusions}

In this paper we focused on the statistics followed by $B_{s}$ waiting-time distributions, at a minimum and at a maximum of the solar activity. By comparing the results obtained, we found differences in the waiting-time statistics, fitting both distributions with a power law plus an exponential cutoff. We found that the power law behavior extends to longer time intervals at solar maximum than at minimum, suggesting that time correlations last longer. At solar minimum, the loss of the long-term correlations is attributed to an enhanced presence of stochastic Alfvén waves (see also Bruno et al., 2003). This conclusion is also supported by the fact that we find a cutoff around the typical hourly period of these fluctuations (Bruno et al., 1985; Marsch and Tu, 1990).

The previous results can be interpreted by considering the solar wind as a superposition of stochastic Alfvénic fluctuations which propagate and structures that are convected by the wind, as suggested by several authors (see review by Bruno and Carbone, 2005). Whether or not Alfvénic fluctuations dominate over convected structures, the waiting-time statistics of $B_{s}$ results to be more or less Poissonian.

We found that these statistics strongly depend on different phases of the solar activity cycle, since the Alfvénic content of solar wind fluctuations changes. During solar minimum, corotating high velocity streams dominate the solar wind dynamics, and associated Alfvénic fluctuations tend to destroy long-term correlations in the waiting-time statistics. On the contrary, during solar maximum, the presence of Alfvénic fluctuation is reduced, and convected structures start to influence the waiting-time statistics with their long-term correlations.

We wonder whether or not the differences found in the waiting-time statistics for different phases of the solar cycle may influence the magnetospheric fluctuations and affect them in the same way. This will be the starting point of future investigations in the context of Sun-Earth connections.

Acknowledgements. We are grateful to the following people and organizations for the provision of data used in this study: R. P. Lepping and NASA Goddard Space Flight Center for WIND MFI data; N. Ness and the Bartol Research Institute for ACE MFI data and F. Mariani and N. Ness for Helios 2 GSFC magnetic experiment. Last but not least, the authors would like to thank the referee for his suggestions and comments.

Topical Editor B. Forsyth thanks M. Freeman for his help in evaluating this paper. 


\section{References}

Bavassano, B., Dobrowolny, M., Fanfoni, G., Mariani, F., and Ness, N. F.: Statistical properties of MHD fluctuations associated with high-speed streams from Helios-2 observations, Solar Phys., 78, 373-384, 1982.

Boffetta, G., Carbone, V., Giuliani, P., Veltri, P., and Vulpiani, A.: Power laws in solar flares: self-organized criticality or turbulence?, Phys. Rev. Lett., 83, 4662-4665, 1999.

Bruno, R., Bavassano, B., and Villante, U.: Evidence for long period Alfvén waves in the inner solar system, J. Geophys. Res., 90(A5), 4373-4377, 1985.

Bruno, R., Carbone, V., Sorriso-Valvo, L., and Bavassano, B.: Radial evolution of solar wind intermittency in the inner heliosphere, J. Geophys. Res., 108(A3), SSH 8-1, 2003.

Bruno, R., Carbone, V., Primavera, L., Malara, F., Sorriso-Valvo, L., Bavassano, B., and Veltri, P.: On the probability distribution function of small-scale interplanetary magnetic field fluctuations, Ann. Geophys., 22, 3751-3769, 2004, http://www.ann-geophys.net/22/3751/2004/.

Bruno, R. and Carbone, V.: The Solar Wind as a Turbulence Laboratory, Living Rev. Solar Phys., 2, 4-186, 2005.

D'Amicis, R., Bruno, R., and Villante, U.: A comparative study of probability distribution functions and burst lifetime distributions of $B_{S}$ and $\mathrm{AE}$ at solar maximum and minimum, Multiscale Coupling of Sun-Earth Processes, edited by: Lui, A. T. Y., Kamide, Y., and Consolini, G., Elsevier Publishing, 399-409, 2004.

Freeman, M. P., Watkins, N. W, and Riley, D. J.: Evidence for a solar wind origin of the power law burst lifetime distribution of the AE indices, Geophys. Res. Lett., 27(8), 1087-1090, 2000a.

Freeman, M. P., Watkins, N. W., and Riley, D. J.: Power law distributions of burst duration and interburst interval in the solar wind: Turbulence or dissipative delf-organized criticality?, Phys. Rev. E, 62(6), 8794-8797, 2000b.

Frisch, U.: Turbulence, The legacy of A. N. Kolmogorov, Cambridge University Press, 120-194, 1995.
Hnat, B., Chapman, S. C., Rowlands, G., Watkins, N. W., and Freeman, M. P.: Scaling of solar wind $\epsilon$ and the AU, AL and AE indices as seen by WIND, Geophys. Res. Lett., 29(10), 35-1, doi:10.1029/2002GL016054, 2002.

Hnat, B., Chapman, S. C., Rowlands, G., Watkins, N. W., and Freeman, M. P.: Correction to "Scaling of solar wind $\epsilon$ and the AU, AL and AE indices as seen by WIND", Geophys. Res. Lett., 30(10), 6-1, doi:10.1029/2003GL017194, 2003.

Hnat, B., Chapman, S. C., and Rowlands, G.: Scaling and a FokkerPlanck model for fluctuations in geomagnetic indices and comparison with solar wind $\epsilon$ as seen by Wind and ACE, J. of Geophys. Res., 110(A9), 8206, doi:10.1029/2004JA010824, 2005.

Marsch, E. and Tu, S.: On the radial evolution of MHD turbulence in the inner heliosphere, J. Geophys. Res., 95, 8211-8229, 1990.

Marsch, E. and Lui, S.: Structure functions and intermittency of velocity fluctuations in the inner solar wind, Ann. Geophys., 11(4), 227-238, 1993.

Tsurutani, B. T. and Gonzalez, W. D.: The future of geomagnetic storm predictions: implications from recent solar and interplanetary observations, J. Atmos. Solar-Terr. Phys, 57(12), 13691384, 1995.

Tu, C.-Y. and Marsch, E.: A model of solar wind fluctuations with two components: Alfvén waves and convective structures, J. Geophys. Res., 98, 1257-1276, 1993.

Tu, C.-Y. and Marsch, E.: MHD structures, waves and turbulence in the solar wind: Observations and theories, Space Sci. Rev., 73, $1-210,1995$.

Vasyliunas, V. M.: Theoretical models of magnetic field line merging, I., Rev. Geophys. and Space Phys., 13, 303-336, 1975.

Vörös, Z., Jankovičová, D., and Kovács, P.: Scaling and singularity characteristics of solar wind and magnetospheric fluctuations, Nonlin. Processes Geophys., 9, 149-162, 2002, http://www.nonlin-processes-geophys.net/9/149/2002/. 